\title{
Antibiotic Susceptibility Profile of Group B Streptococcus (Streptococcus agalactiae) at the Maternity Hospital, Kuwait
}

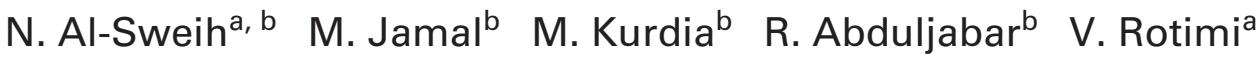 \\ ${ }^{a}$ Department of Microbiology, Faculty of Medicine, Kuwait University and ${ }^{b}$ Maternity Hospital, Kuwait
}

\section{Key Words}

Group B streptococcus · Susceptibility · Intrapartum chemoprophylaxis

\begin{abstract}
Objectives: This study was designed to determine the antibiotic susceptibility profile of clinical isolates of group B streptococcus (GBS, Streptococcus agalactiae) and to use the information for formulating appropriate intrapartum antibiotic policy for GBS carriage in pregnancy. Materials and Methods: A total of 1,166 clinical isolates (single isolates) of GBS obtained from vaginal/ rectal samples of pregnant mothers seen at the Maternity Hospital, Kuwait were studied over a period of 43 months between May 1998 and December 2001. The isolates were identified by standard methods and their susceptibility to penicillin, ampicillin, cephalothin, clindamycin and erythromycin was determined by disk diffusion technique, minimal inhibitory concentration (MIC) using the Vitek automated sensitivity card system and E-test methods. Results: All the GBS isolates were fully susceptible to penicillin, ampicillin and cephalothin. Only 0.7 and $1.7 \%$ were resistant to erythromycin and clindamycin, respectively. Disk diffusion results interpreted by the standard interpretative criteria recommended by the National Committee on Clinical Laboratory Standards correlated well with Vitek results as well as the E-test for penicillin. The MIC of penicillin against all isolates ranged between 0.016 and $0.064 \mu \mathrm{g} / \mathrm{ml}$. For
\end{abstract}

the 6 months of 1998 and throughout 1999, the percentages of isolates susceptible at MICs of $0.016,0.023,0.032$, 0.047 and $0.064 \mu \mathrm{g} / \mathrm{ml}$ were $6.5,9.9,31,38.8$ and $12 \%$, respectively. The trend was similar in the subsequent years except that the percentage of isolates susceptible at MIC of $0.064 \mu \mathrm{g} / \mathrm{ml}$ increased to $26.6 \%$ in 2000 , but went down to $4.4 \%$ in 2001. Conclusion: The trend in susceptibility of GBS to a variety of often used antibiotics for therapy and prophylaxis remained unchanged over nearly a 4-year period. The apparent increase in the number of isolates susceptible at higher MIC values of penicillin $(0.047$ and $0.064 \mu \mathrm{g} / \mathrm{ml})$ in 2000 appears to be a bleb that cannot be explained by any event in the hospital for that year. Our data, based on susceptibility profiles, supports the use of penicillin or ampicillin for intrapartum chemoprophylaxis to prevent early-onset neonatal GBS infections.

Copyright $@ 2005$ S. Karger AG, Basel

\section{Introduction}

Streptococcus agalactiae, also known as group B streptococcus (GBS), is a frequent cause of serious infections often associated with mortality and morbidity in newborn babies [1-3]. Neonates born of vaginally colonized mothers stand a great risk of acquiring the organism from the birth canal during delivery. Acquisition of GBS by neonates can lead to two different spectra of infections recognized as early-onset or late-onset diseases. Preven-

\section{KARGER \\ Fax +4161306 1234 E-Mail karger@karger.ch} www.karger.com
(C) 2005 S. Karger AG, Basel

$1011-7571 / 05 / 0144-0260 \$ 22.00 / 0$

Accessible online at:

www.karger.com/mpp
Dr. Noura A. AL-Sweih, MD, FRCPath

Department of Microbiology, Faculty of Medicine, Kuwait University PO Box 24923

13110 Safat (Kuwait)

Tel./Fax +965 5332719, E-Mail nalsweih@hsc.edu.kw 
tion of neonatal colonization during labor is therefore thought by many experts to be worthwhile. The body of evidence in the literature shows that intrapartum chemoprophylaxis is an effective means of preventing vertical transmission of GBS $[4,5]$. A number of studies have demonstrated a significant reduction of early-onset neonatal infections by intrapartum chemoprophylaxis with penicillin or ampicillin in patients colonized with GBS [6-9]. A major worry among the obstetricians concerns administration of beta-lactam antibiotics to women with penicillin allergy. Thus, the Center for Disease Control, USA (CDC) protocol recommends clindamycin or erythromycin as suitable alternatives [9].

The comparative efficacy and susceptibility of GBS to other agents have been assessed infrequently [10, 11]. Previous reports have documented universal susceptibility of GBS to penicillin, and consensus guidelines for the prevention of early-onset neonatal GBS disease do not call for antimicrobial susceptibility testing of GBS isolates to guide antibiotic prophylaxis [12]. However, with the widespread use of intrapartum antibiotics (mainly ampicillin in our hospital) for prophylaxis against vaginal carriage of GBS in pregnancy and neonatal infections, there is a potential for the emergence of resistant GBS strains. Therefore, the present study was undertaken to determine the incidence of antibiotic-resistant clinical isolates of GBS, isolated from genitourinary and other sites.

\section{Materials and Methods}

All consecutive GBS isolates from clinical specimens, such as high and low vaginal swabs, urine, wound, blood, cerebrospinal fluid and miscellaneous sources were studied over a period of 4 years between January 1998 and December 2001. Single isolate per site was collected from each individual. The isolates were identified as GBS on the basis of being catalase-negative, gram-positive cocci and giving appropriate reaction with commercial latex-group-specific streptococcal typing system (Slidex Strepto, BioMérieux SA, Lyon, France) and Vitek identification system (BioMérieux). The strains were tested in batches following the same procedures and ensuring consistency in protocol. Isolates were tested for antimicrobial susceptibility to penicillin, ampicillin, cephalothin, clindamycin and erythromycin by Kirby-Bauer disk diffusion susceptibility testing method. Briefly, bacteria grown on blood agar (Oxoid, Basingstoke, UK) supplemented with 5\% sheep were resuspended in sterile saline to a $0.5 \mathrm{McFarland}$ turbidity standard as an inoculum containing $10^{5} \mathrm{cfu} / \mathrm{ml}$. This was inoculated onto a MuellerHinton agar plate, by streaking evenly with a swab in three directions over the entire surface to obtain a uniform lawn of growth. The plates were allowed to dry for 3-5 min and the antibiotic disks carefully placed, at equidistance from each other, on the inoculated agar plates. Then the plates were incubated in an aerobic incubator
Table 1. Frequent sites of isolation of group B streptococcus

\begin{tabular}{lc}
\hline Site & $\mathrm{n}(\%)$ \\
\hline Vagina & $821(70.4)$ \\
Urine & $225(21.9)$ \\
Wound & $28(2.4)$ \\
Blood & $11(0.9)$ \\
Cerebrospinal fluid & $1(0.1)$ \\
Miscellaneous & $50(4.3)$ \\
Total & $1,166(100)$ \\
\hline
\end{tabular}

at $35^{\circ} \mathrm{C}$ for $24 \mathrm{~h}$. The zone sizes were then recorded for each antimicrobial agent and interpreted according to the National Committee for Clinical Laboratory Standards (NCCLS) [13] interpretative criteria for streptococci.

In parallel, at the same time, the antimicrobial susceptibility of all isolates was also tested using an automated Vitek IS card system (BioMérieux) using the same panel of antibiotics following the manufacturer's instruction. The inoculum used was the same as for the disk diffusion test.

In addition to the two methods described above, the minimal inhibitory concentrations (MICs) of penicillin and ampicillin were the same standardized inoculum. Interpretation of the results was according to the manufacturer's instructions. The MICs obtained were categorized as susceptible, intermediate or resistant based on the antibiotic breakpoint concentrations recommended by the NCCLS [13].

\section{Results}

A total of 1,166 GBS isolates were collected during this study period; 207 in 1998, 311 in 1999, 327 in 2000 and 321 in 2001. As shown in table 1, the distribution of the isolates by site indicated that the majority were from vaginal swabs, representing $70.4 \%$, followed by urine, $22 \%$. All of the 1,166 clinical isolates tested were fully susceptible to penicillin, ampicillin and cephalothin. Only 8 $(0.7 \%)$ and $20(1.7 \%)$ were resistant to erythromycin and clindamycin, respectively. The susceptibility tests by the three different methods (disk diffusion, automated Vitek and E-test) revealed no significant difference in the interpretation of the results. There were no discrepant results and the E-test MIC results for penicillin were concordant with both disk diffusion and Vitek results. An analysis of the data generated by the E-test for penicillin MIC determination showed no incidence of penicillin-resistant isolate.

As demonstrated in table 2, the MIC range of penicillin in the 4 years' study period was $0.016-0.064 \mu \mathrm{g} / \mathrm{ml}$. 
Table 2. Susceptibility of GBS to penicillin by MIC determination

\begin{tabular}{llrrrr}
\hline \multirow{2}{*}{ Year } & \multicolumn{5}{c}{$\%$ of isolates susceptible to penicillin at $\mathrm{MIC}(\mu \mathrm{g} / \mathrm{ml})$ of } \\
\cline { 2 - 6 } & 0.016 & 0.023 & 0.032 & 0.047 & 0.064 \\
\hline 1998 & 7.2 & 10.6 & 31.4 & 38.6 & 12.1 \\
1999 & 7.4 & 10.3 & 31.2 & 38.9 & 12.2 \\
2000 & 0.6 & 1.8 & 30.6 & 40.4 & 26.6 \\
2001 & 5.0 & 23.0 & 47.7 & 19.9 & 4.4 \\
\hline
\end{tabular}

The percentages of susceptible isolates clustering at MIC of $0.064 \mu \mathrm{g} / \mathrm{ml}$ (susceptibility breakpoint) were 12.1, $12.2,26.6$ and $4.4 \%$ in $1998,1999,2000,2001$, respectively, with an overall rate of $14.1 \%$ of the 1,166 isolates having increased MIC value.

\section{Discussion}

In this study, all the antibiotics tested had excellent in vitro activities against the clinical isolates of GBS. The trend in their susceptibility was essentially similar during the 4-year study period. This is noteworthy as the efficacy of any antimicrobial chemoprophylaxis strategy is influenced strongly by the susceptibility of the targeted microorganism to the agent employed. Most of the isolates in this study were isolated from genitourinary sites and such isolates are almost without exception the only ones that can cause an early-onset GBS neonatal disease. Moreover, the isolates were obtained from our hospital where guidelines of the American Collage of Obstetric and Gynecology Risk-Factor-based approach for prevention of early-onset neonatal GBS infection had been used consistently before the collection of GBS specimens [14].

Thus this investigation offered the potential to detect emerging resistance pattern resulting from the implementation of an early-onset neonatal GBS prevention strategy. The continued susceptibility of GBS to members of the penicillin family, with MICs that should be readily achieved in fetal blood using the standard dosing regimens $[15,16]$, plus the resistance of few isolates to other antibiotics, support the first-line role of penicillin and ampicillin in prophylactic treatment regimens against earlyonset neonatal GBS disease. Because of its narrow spectrum of action, and therefore decreased potential for the selection of resistant organisms, penicillin $\mathrm{G}$ may be preferable to ampicillin. On the other hand, ampicillin crosses the placenta more quickly than penicillin $\mathrm{G}$ and achieves high concentrations in the fetal compartment $[15,16]$. However, comparative clinical data on the efficacy of penicillin $G$ and ampicillin for the prevention of early-onset neonatal GBS disease is lacking.

Clindamycin and erythromycin are recommended currently as second-line antimicrobial for intrapartum chemoprophylaxis for GBS infections for women who are allergic to penicillin $[9,14]$. Resistance of GBS to clindamycin and/or erythromycin has already been reported, ranging from 3 to $16 \%$ for erythromycin and from 2 to $15 \%$ for clindamycin $[10,11,17,18]$. It is worthy of note that the resistance rates to these two antibiotics $(0.7 \%$ for erythromycin and $1.7 \%$ for clindamycin) recorded in this study appear to be among the lowest so far encountered [10-12, 17-20], thus suggesting that the recommended alternative antibiotic in the CDC protocol is amenable to adoption in our environment and conceivably providing options for reducing the risk of neonatal GBS infection in penicillin-allergic mothers. In addition, in such circumstances patients at risk of preterm labor who are colonized with GBS can also be adequately treated.

With the low rate of resistance to erythromycin in our study, erythromycin may be the better alternative to penicillin; however, clindamycin may still be preferable to erythromycin because it more readily and consistently crosses the placenta and achieves higher fetal concentrations than erythromycin [21, 22]. The efficacy of either agent for eradicating maternal colonization, decreasing vertical transmission of GBS or preventing GBS disease in neonates at the intrapartum doses recommended currently was not evaluated and there are very few anecdotal reports that support these clinical observations [14].

These limitations raise serious reservations about selection of these agents as alternatives to penicillin for GBS prophylaxis. The use of potentially ineffective alternatives to penicillin could probably be reduced by careful history concerning penicillin allergy or using first-generation cephalosporins which share many of pharmacokinetic properties of penicillin. All isolates in this study were fully susceptible to cephalothin, but their ability to prevent vertical transmission of GBS and early-onset disease has not been studied, and, importantly, about $8 \%$ of patients with severe penicillin allergy may manifest crosshypersensitivity to penicillin [23].

Even though all isolates were susceptible to penicillin, a fairly large proportion (approximately 14\%) exhibited decreased susceptibility, being only inhibited at an MIC value just a mode dilution below the cutoff point for intermediate resistance. This is a significant observation 
worthy of constant monitoring as this may be a precursor to the emergence of true penicillin resistance in GBS. Routine susceptibility testing of clinically significant GBS isolates is therefore recommended. Our study has shown that both disk diffusion test and Vitek automated method produce the same results as the E-test, which can be expensive for some laboratories. Vitek system is available in all laboratories in Kuwait and, in our hands, has proven to be reliable and cost-effective.

\section{Conclusion}

Our data confirmed the universal susceptibility of GBS to the penicillin family and supports the use of penicillin or ampicillin for intrapartum chemoprophylaxis to prevent early-onset neonatal GBS infections. For patients allergic to penicillin, clindamycin or erythromycin may be used as an alternative since these drugs have very little adverse effects on pregnancy in the 3rd trimester.

\section{References}

1 Baker CJ: Group B streptococcal infections. Infect Perinatol 1997;24:59-70.

-2 Zangwill KM, Schuchat A, Wenger JD: Group B streptococcal disease in the United States, 1990: Report from a multistate active surveillance system. MMWR Morb Mortal Wkly Rep 1992;41:25-32.

3 American College of Obstetricians and Gynecologists: Group B Streptococcal Infections in Pregnancy. Washington, ACOG, 1992. ACOG Techn Bull No 170.

-4 Garland SM, Fliegner JR: Group B streptococcus and neonatal infections: The case for intrapartum chemoprophylaxis. Aust NZJ Obstet Gynaecol 1991;31:119-122.

5 Levine EM, Ghai V, Barton JJ, Storm CM: Intrapartum antibiotic prophylaxis increases the incidence of gram-negative neonatal sepsis. Infect Dis Obstet Gynecol 1986;7:210-213.

-6 Yow MD, Mason EO, Leeds LJ, Thompson PK, Clark DJ, Gander SE: Ampicillin prevents intrapartum transmission of group B streptococcus. JAMA 1979;241:1245-1247.

7 Lim DV, Morales WJ, Walsh AF, Kazanis D: Reduction of morbidity and mortality rates of neonatal group B streptococcal disease through early diagnosis and chemoprophylaxis. J Clin Microbiol 1986;23:489-492.

-8 Boyer KM, Gotoff SP: Prevention of early-onset neonatal group B streptococcal disease with selective intrapartum chemoprophylaxis. N Engl J Med 1986;314:1665-1669.
-9 Center for Disease Control: Prevention of perinatal group B streptococcus disease: A public health perspective. MMWR Morb Mortal Wkly Rep 1996;45(RR-7):1-24.

10 Berkowitz K, Regan JA, Greenberge E: Antibiotic resistance patterns of group B streptococci in pregnant women. J Clin Microbiol 1990;28: 5-7.

11 Betriu C, Gomez M, Sanchez A, Cruceyra A, Romero J, Picazo J: Antibiotic resistance and penicillin tolerance in clinical isolates of group B streptococci. Antimicrob Agents Chemother 1994;38:2183-2186.

12 Arora A, Jindal N, Walia G, Guati VL: Presumptive identification and antibiotic susceptibility of group B streptococci. Indian J Pathol Microbiol 1994;38:185-190.

13 National Committee for Clinical Laboratory Standards: Performance Standards for Antimicrobial Susceptibility Testing: Eighth Informational Supplement, vol 18, No 1. Wayne, National Committee for Clinical Laboratory Standards, 1998.

14 American College of Obstetricians and Gynecologists: Prevention of early-onset group B streptococcal disease in newborns. ACOG Committee Opinion No 173. Washington, American College of Obstetricians and Gynecologists, 1996.

15 Neu H: Clinical pharmacokinetics in pregnancy and perinatology. II. Penicillins. Dev Pharmacol Ther 1987;10:174-198.
16 Was Z, Hockerl O, Nummi S, Vuopala S, Jarvinen PA: Transplacental passage of azidocillin, ampicillin and penicillin G during early and late pregnancy. Scand J Infect Dis 1970;2: 125-130.

17 Baker CJ, Webb BJ, Barrett FF: Antimicrobial susceptibility of group B streptococci isolated from a variety of clinical sources. Antimicrob Agents Chemother 1976;10:128-131.

18 Tsakris A, Maniatis AN: Inducible type of erythromycin resistance among group B streptococci isolated in Greece. J Antimicrob Chemother 1993;31:177-178.

19 Fernandez M, Hickman ME, Baker CJ: Antimicrobial susceptibilities of group B streptococci isolated between 1992 and 1996 from patients with bacteremia or meningitis. Antimicrob Agents Chemother 1998;42:15171519.

20 Pearlman MD, Pierson CL, Faix RG: Frequent resistance of clinical group B streptococci isolates to clindamycin and erythromycin. Obstet Gynecol 1998;92:256-261.

-21 Philipson A, Sabath LD, Charles D: Transplacental passage of erythromycin and clindamycin. N Engl J Med 1973;288:1219-1221.

22 Committee on Infectious Diseases: $1997 \mathrm{Red}$ Book: Report of the Committee on Infectious Diseases, ed 24. Elk Grove Village, American Academy of Pediatrics, 1997, pp 504-514.

23 Petz LD: Immunologic cross-reactivity between penicillin and cephalosporins: A review. J Infect Dis 1978;137:S74-S79. 